\title{
Successful optical coherence tomography-guided stent ablation with rotational atherectomy for an underexpanded stent
}

\author{
Yongcheol Kim®, Deok-Kyu Cho®, Ji Woong Roh®, \\ Oh-Hyun Lee®, Eui Im®, Donghoon Choi® \\ Division of Cardiology, Department of Internal Medicine, Yonsei University College of Medicine \\ and Cardiovascular Center, Yongin Severance Hospital, Yongin, Korea
}

A 62-year-old man with a history of ischemic heart disease and dyslipidemia presented with aggravating effort angina. He underwent stent implantation with a $3.0 \times 40 \mathrm{~mm}$ sirolimus-eluting stent at the mid portion of left anterior descending artery 2 years prior, but a heavily calcified lesion led to severe stent underexpansion, which did not resolve despite postdilation with a non-compliant (NC) balloon (Suppl. Video 1). Angiography demonstrated aggravated luminal narrowing in the underexpanded stent site (Fig. 1A, Suppl. Video 2); therefore, stent ablation with rotational atherectomy was planned. Pre-interventional optical coherence tomography (OCT) demonstrated that the diameter of the underexpanded stent was between 1.24 and $1.66 \mathrm{~mm}$ (Fig. 1B, C, Suppl. Video 3), which was also confirmed on three-dimensional OCT (Fig. 1D). OCT assessment led to stent ablation using a stepwise increase in burr size, from
$1.25 \mathrm{~mm}$ to $1.50 \mathrm{~mm}$ and finally $1.75 \mathrm{~mm}$. Following stent ablation, OCT showed no visible strut area in the site of the previously underexpanded stent (Fig. 1E, F). However, a minimal lumen area of $2.27 \mathrm{~mm}^{2}$ led to the performance of drug-coated balloon angioplasty with a $3.0 \times 20 \mathrm{~mm}$ Pantera Lux (Biotronik, Bülach, Switzerland) after balloon dilation with a $3.0 \times 15 \mathrm{~mm} \mathrm{NC}$ balloon at $24 \mathrm{~atm}$. Final angiography showed good distal flow without residual stenosis (Fig. 1G, Suppl. Video 4).

This report highlights the superior resolution of OCT, which can aid in choosing the optimal burr size, and presents OCT images of successful stent ablation.

\section{Acknowledgements}

The authors would like to thank all the staff, especially Dae Seok Jang, working in the cardiac catheterization laboratories at Yongin Severance Hospital for their commitment to this study.

Conflict of interest: None declared

Address for correspondence: Deok-Kyu Cho, MD, Cardiovascular Center, Yongin Severance Hospital, 363 Dongbaekjukjeon-daero, Giheung-gu, Yongin 16995, Korea, tel: 82-31-5189-8755, fax: 82-31-5189-8567, e-mail: chodk123@yuhs.ac

Received: 5.06.2020 Accepted: 5.06.2020 


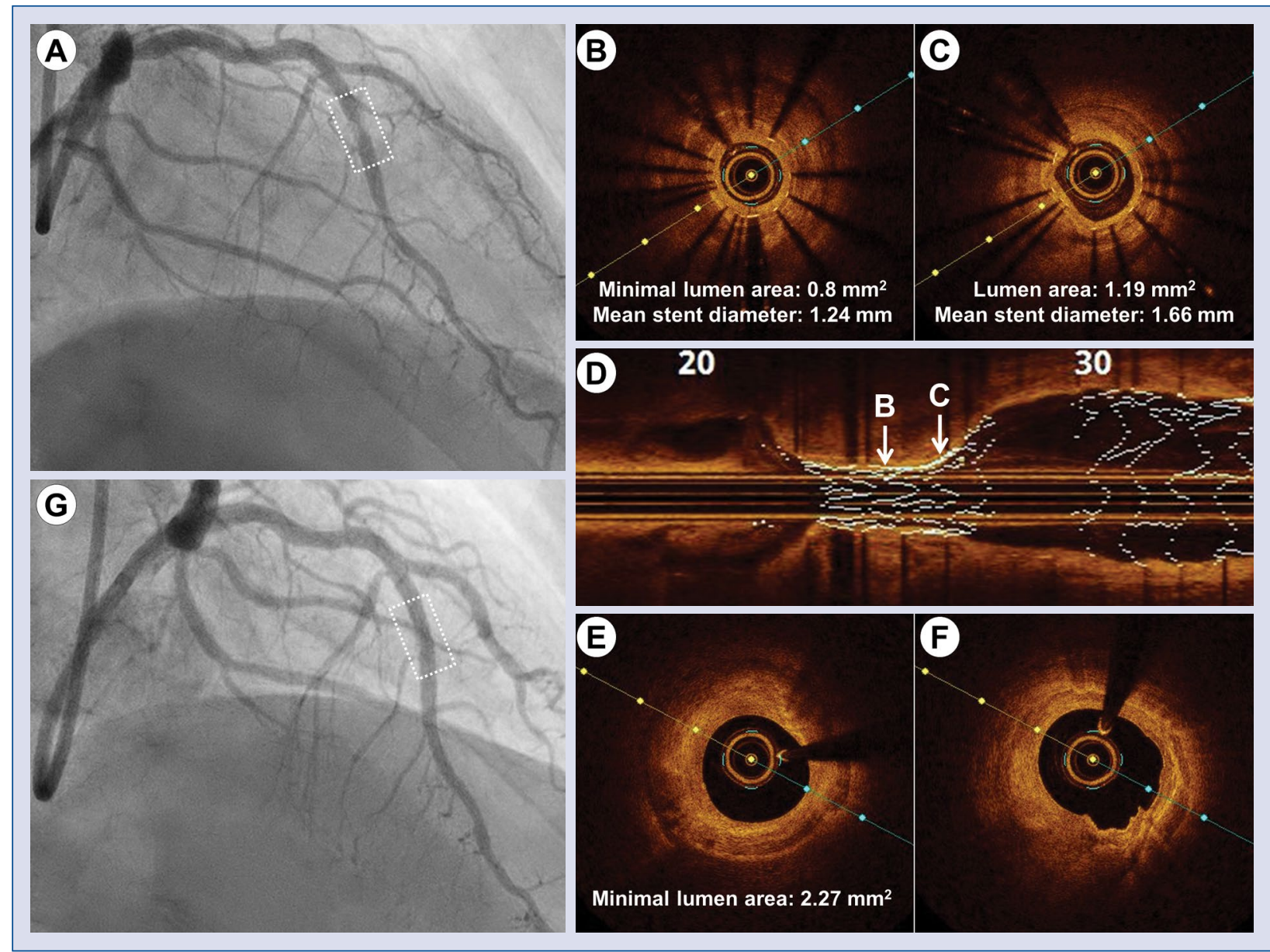

Figure 1. A. Initial angiography demonstrating underexpanded stent (white box); B, C. Cross-sectional optical coherence tomography (OCT) imaging of underexpanded stent site; D. Three-dimensional OCT reconstructed strut image of underexpanded stent site; E, F. OCT demonstrating no visible struts after stent ablation with rotational atherectomy; G. Final angiography demonstrating no residual stenosis after treatment with $3.0 \times 20 \mathrm{~mm}$ drug-coated balloon. 